\title{
The Minimal Clinically Important Difference in Glaucoma Medication Adherence: Interviews of Glaucoma Experts
}

\author{
Ajay Kollia Shelby Daniel-Wayman ${ }^{b}$ Paula Anne Newman-Casey ${ }^{b}$ \\ aUniversity of Michigan Medical School, Ann Arbor, MI, USA; bepartment of Ophthalmology and Visual Sciences, \\ University of Michigan, Ann Arbor, MI, USA
}

\section{Keywords}

Glaucoma - Minimal clinically important difference .

Adherence

\begin{abstract}
Poor adherence to glaucoma medications is associated with progressive vision loss. While many interventions have sought to increase glaucoma medication adherence, the amount by which adherence must increase to have a clinically significant effect remains unknown. To generate a hypothesized minimal clinically important difference (MCID) for glaucoma medication adherence, we conducted interviews with glaucoma experts. Semi-structured interviews were conducted with members of the American Glaucoma Society. MCID was defined in 2 ways: (1) the incremental increase in the average percentage of eye drops a patient takes at roughly the correct time and (2) the incremental increase in the proportion of a patient population who attain good adherence. Good adherence was defined as taking more than $80 \%$ of drops at approximately the prescribed dose time. Expert opinions on the MCID for glaucoma medication adherence and open-ended responses were recorded through field notes. Twenty-five experts were interviewed. They estimated the MCID for average individual adherence levels as $17.7 \%(95 \% \mathrm{Cl}: 14.6,20.8)$. Experts estimated the MCID for the proportion of patients in a practice who attain
\end{abstract}

karger@karger.com

(c) 2020 S. Karger AG, Basel

www.karger.com/ore

Karger $\stackrel{2}{=}$ good adherence (defined as $>80 \%$ of eye drops taken as prescribed) as $18.5 \%(95 \% \mathrm{Cl}: 15.6,21.5)$. The most common identified themes were that the MCID should take into account the cost of the intervention and the burden to the ophthalmologist and to the practice, where experts thought that more costly interventions or those that required more physician time should have larger MCIDs. Based on expert opinion, we hypothesized that the MCID for glaucoma medication adherence is between 15 and 20\%. However, the MCID for a given intervention must take into account several factors, including intervention cost and physician burden. This hypothesis may facilitate the design and implementation of future studies to objectively determine an MCID for glaucoma medication adherence.

(c) 2020 S. Karger AG, Basel

\section{Introduction}

Poor adherence to therapeutic regimens is responsible for substantial morbidity, mortality, and healthcare costs in the USA [1]. In glaucoma, poor adherence has been associated with progressive vision loss $[2,3]$. In a crosssectional survey, Sleath et al. [4] found that patients taking less than $80 \%$ of their prescribed glaucoma medication doses had greater visual field defect severity. In turn, recent studies have defined "good adherence" as taking 
greater than $80 \%$ of prescribed eye drops [5]. Developing and testing interventions to improve glaucoma medication adherence has become an important target of research [6]. However, no studies have quantified what incremental improvement in medication adherence is necessary to stabilize visual field loss.

There is no consensus regarding what indicates success for interventions that aim to improve medication adherence. In 2018, the European Society for Patient Adherence, Compliance, and Persistence guidelines made recommendations for reporting trial information about medication adherence interventions [7]. However, these new guidelines do not delineate a definition of success for adherence interventions. This may, in part, be because success must be defined separately for each independent disease state. The minimal clinically important difference (MCID) refers to the smallest amount an outcome (e.g., percentage of eye drops used or proportion of patients who have good adherence) must change to have a significant impact on a patient's clinical course [8]. One Cochrane review suggested an overarching MCID of 25\% and further went on to define MCIDs for different disease states based on expert clinician opinion [9]. For example, MCIDs of 5 and 20\%, respectively, were suggested for COPD and osteoporosis [9]. These suggested MCIDs were based on expert opinion, given the lack of objective, biologic data to inform the MCID for each different disease [9-12].

The MCID for glaucoma medication adherence remains unknown. To address this gap, we conducted semistructured interviews with glaucoma experts to generate a hypothesized MCID for glaucoma medication adherence. In turn, this hypothesized MCID may facilitate future studies that aim to determine an appropriate MCID using quantitative, biological outcomes.

\section{Materials and Methods}

Interview participants were recruited from a convenience sample of 66 members of the American Glaucoma Society e-mail list serve who had participated in a previous, unpublished surveybased study regarding medication adherence. Participants were recruited by asking if they were willing to provide their contact information in order to participate in an in-person interview at the annual meeting of the American Glaucoma Society Participants included fellowship-trained glaucoma specialists, fellows in glaucoma training, and scientists involved in glaucoma research. Participant years since fellowship graduation and practice location were collected to evaluate sample diversity. Participants were aware that the goal of this research was to determine a hypothesized MCID for glaucoma medication adherence. The University of Michigan Institutional Review Board determined that this study was exempt of IRB approval and formal written consent was not required. Verbal consent was obtained from all participants.

Participants who provided their contact information were contacted by the investigator (PANC), an academic glaucoma specialist, and scheduled for a semi-structured, in-person, 10-min interview to offer their expert opinion on an appropriate MCID for glaucoma medication adherence and offer open-ended comments. "Change in adherence" was defined in 2 separate ways: (1) the percent of drops taken at approximately the prescribed time each month [8] and (2) the proportion of patients in a practice that are adherent to their medications [5]. In the latter definition, patients were considered to be adherent if they take greater than $80 \%$ of their drops at approximately the prescribed time [4, 5]. For each of these definitions of adherence, participants were asked to estimate what percentage increase in adherence would likely impact clinical outcomes. Mean MCIDs and 95\% confidence intervals (CIs) were calculated from individual participant responses. After initial response, participants were asked an open-ended question encouraging further elaboration, and field notes were taken (PANC) for analysis.

The field notes were analyzed using a grounded theory approach, an inductive process of generating theories, beginning with qualitative data $[13,14]$. The 2 coders (PANC and AK) followed a stepwise approach typical of GT: (1) familiarization, (2) open coding, (3) focused coding, and (4) theory building. Any discrepancies in coding between the 2 coders were resolved via consensus. An academic glaucoma specialist (PANC) conducted the interviews and generated field notes. Representative quotes were selected for presentation for each theme in the manuscript based on their representativeness and depth of contextual information.

\section{Results}

Of 66 individuals who completed the survey and were contacted to participate in the present study, 29 (44\% response rate) agreed to participate in a semi-structured interview and 25/29 participated (14\% attrition). Participants had a median fellowship graduation year of 2004 (interquartile range: 1991-2014) and practiced in 18 different states in the US and Australia. Thematic saturation was reached after interviewing 12 participants, similar to what is suggested in the qualitative literature [15].

Experts estimated the MCID for average percent of drops taken at approximately the correct time each month as $17.7 \%(95 \% \mathrm{CI}=14.6,20.8)$. In other words, if participants took $50 \%$ of eye drops at the correct time prior to an intervention, and this improved to $67.7 \%$ after intervention, then the intervention would be considered clinically successful. Experts estimated the MCID for the proportion of patients in a practice who attain good adherence $(>80 \%$ adherence $)$ at a mean of $18.5 \%(95 \% \mathrm{CI}=$ $15.6,21.5)$. For example, if $50 \%$ of patients in an ophthalmology practice had "good adherence" prior to an intervention, and this increased to $68.5 \%$ after intervention, 
then the intervention would be considered clinically successful.

The most commonly identified qualitative theme was that the MCID should take into account the intervention cost and burden to the practice and the physician. For example, 1 participant stated that he was "worried about the burden of so many visits and costs for patients, so I would only implement it in my practice if it improved adherence for $25 \%$ of patients." However, he further stated, "if a paraprofessional staff member was doing the counseling and insurance paid for it, I would implement it if it improved adherence for $15 \%$ of my patients." Other ophthalmologists agreed that practice burden and intervention cost would modulate the MCID for any given intervention. Participants who offered similar insights suggested an MCID of 20-25\% for interventions with high cost or practice burden, but $5-15 \%$ for less costly or physician time-intensive interventions. One participant stated that if an intervention "was not slowing down practice flow, then I would want it if it even improved adherence for $5 \%$ of my patients." Another participant agreed that "even a small change in adherence might be really important" because a " $1 \mathrm{~mm} \mathrm{Hg}$ reduction in IOP leads to a $10 \%$ reduction in relative risk of glaucoma progression" [16].

Other experts also suggested a single MCID for glaucoma medication adherence may not capture all clinically important information, and a more nuanced approach may be needed. For example, patients with a new diagnosis of glaucoma may have a different MCID than those who have already established care. Additionally, another participant suggested that the MCID should not be used as the sole measure of an intervention's success. She stated, "the impact on patient satisfaction and patient reported outcome measures is also important" [17].

\section{Discussion}

Despite the availability of effective treatments, glaucoma remains the second leading cause of blindness in the USA $[16,18,19]$. As with other conditions, rates of poor adherence are high, and patients with worse adherence have worse glaucoma-related vision loss [3, 4]. Historically, "good adherence" to medication has been defined as taking at least $80 \%$ of prescribed doses over a specified time period. This standard was introduced in 1982 by researchers studying adherence to antihypertensive medications [20]. In turn, many more recent studies define adequate compliance as $80 \%$, within and outside the scope of hypertension $[21,22]$. However, this value cannot be universally applied. For example, in HIV, adherence rates of $95 \%$ are considered necessary for adequate disease control [23].

Glaucoma researchers have typically defined "good adherence" as taking $80 \%$ of prescribed medications, due to both this historical definition and studies demonstrating an association between greater than $80 \%$ adherence and relative preservation of visual fields $[4,5,21]$. While many interventions have sought to improve glaucoma medication adherence, the amount adherence must increase to significantly impact clinical care remains unknown. This study serves as a starting point for addressing this knowledge gap by using expert opinion to generate a hypothesized MCID for glaucoma medication adherence.

Experts estimated a mean MCID of $19 \%$ as appropriate for assessing a meaningful improvement in the proportion of patients who attain $80 \%$ adherence. Experts estimated a mean MCID of $18 \%$ as appropriate for assessing a meaningful improvement in mean percentage of drops taken at approximately the correct time each day. From this data, we hypothesize that the MCID for glaucoma medication adherence is between 15 and 20\%. This hypothesized MCID of 15-20\% may facilitate the design and implementation of future studies to objectively determine the MCID. In turn, a biologically defined MCID can also be used in patient education. For instance, an objectively determined MCID would provide patients with a specific and actionable goal for improving their medication adherence.

Subjective analysis of interviews revealed an overarching theme: glaucoma experts suggested that the details of the intervention modulate their estimation of the MCID for glaucoma medication adherence. Specifically, participants suggested that studies of an expensive or time-consuming intervention that disrupts normal workflow should use a larger MCID, in the $20-25 \%$ range. Conversely, for a low-cost intervention or an intervention reimbursed by insurance that can be seamlessly integrated into current practice because the care is delivered by a nonphysician provider, an MCID as low as $5 \%$ may be appropriate in order to bring benefit to more patients.

As elicited in the interviews, a single value for an MCID for glaucoma medication adherence may not be appropriate for every circumstance. Additionally, future work may need to delineate individualized MCIDs for different classes of ocular hypotensive medications as different levels of adherence may lead to differential control of intraocular pressure depending on the pharmacokinetics of 
the medication. Studies using quantitative methods and biological outcomes are needed to more precisely determine the relationship between incremental improvements in glaucoma medication adherence - overall and by medication class - and its impact on intraocular pressure control and visual field loss.

This study has several limitations and aims only to generate a hypothesis regarding an MCID for glaucoma medication adherence. This study used a convenience sample of glaucoma specialists and presents the aggregated opinions of 25, mainly US based, experts on an MCID for glaucoma medication adherence. Thus, this sample may not reflect the entire population of clinicians who care for glaucoma patients, including comprehensive ophthalmologists, optometrists, and primary care physicians. However, prior work has demonstrated the importance of using expert opinion to determine what clinicians' value regarding research and health service outcomes [9-12, 24]. The hypothesis generated in this study should be tested in future studies utilizing quantitatively measured biological outcomes.

\section{Conclusion}

From expert interviews, we hypothesized that an appropriate MCID for glaucoma medication adherence is between 15 and 20\%. Several factors, including intervention costs, may modulate the appropriate MCID for a giv- en intervention. Further research to test, this hypothesis will allow for more meaningful evaluation of the efficacy of glaucoma medication adherence interventions.

\section{Statement of Ethics}

The University of Michigan Institutional Review Board determined that this study was exempt of IRB approval, and formal written consent was not required. Verbal consent was obtained from all participants.

\section{Conflict of Interest Statement}

The authors have no conflicts of interest to disclose.

\section{Funding Sources}

This study was supported by the National Eye Institute (K23EY025320, PANC) and Research to Prevent Blindness Career Development Award (PANC). The funding organizations had no role in the design or conduct of this research.

\section{Author Contributions}

Each author has made substantial contributions to the design, analysis, and interpretation of the work, as well as the drafting of the manuscript. All authors gave final approval for the work.

\section{References}

1 Cutler DM, Everett W. Thinking outside the pillbox: medication adherence as a priority for health care reform. N Engl J Med. 2010; 362(17):1553-5.

2 Rossi GC, Pasinetti GM, Scudeller L, Radaelli $\mathrm{R}$, Bianchi PE. Do adherence rates and glaucomatous visual field progression correlate? Eur J Ophthalmol. 2011;21(4):410-4.

3 Newman-Casey PA, Niziol LM, Gillespie BW, Janz NK, Lichter PR, Musch DC. The association between medication adherence and visual field progression in the collaborative initial glaucoma treatment study. Ophthalmology. 2020;127(4):477-83.

4 Sleath B, Blalock S, Covert D, Stone JL, Skinner AC, Muir K, et al. The relationship between glaucoma medication adherence, eye drop technique, and visual field defect severity. Ophthalmology. 2011;118(12):2398-402.
5 Cate H, Bhattacharya D, Clark A, Fordham R, Holland R, Broadway DC. Improving adherence to glaucoma medication: a randomised controlled trial of a patient-centred intervention (the norwich adherence glaucoma study). BMC Ophthalmol. 2014;14(1):32.

6 PA-18-722: Improving Patient Adherence to Treatment and Prevention Regimens to Promote Health (R01 Clinical Trial Optional) [Internet]. National Institutes of Health. U.S. Department of Health and Human Services; [cited 2020 Jul 17]. Available from: https:// grants.nih.gov/grants/guide/pa-files/pa-18722.html.

7 De Geest S, Zullig LL, Dunbar-Jacob J, Helmy R, Hughes DA, Wilson IB, et al. ESPACOMP medication adherence reporting guideline (EMERGE). Ann Intern Med. 2018;169(1):30.

8 Mcglothlin AE, Lewis RJ. Minimal clinically important difference: defining what really matters to patients. JAMA. 2014;312(13): 1342.
9 Nieuwlaat R, Wilczynski N, Navarro T, Hobson N, Jeffery R, Keepanasseril A, et al. Interventions for enhancing medication adherence. Cochrane Database Syst Rev. 2014(11): CD000011.

10 Normansell R, Kew KM, Stovold E. Interventions to improve adherence to inhaled steroids for asthma. Cochrane Database Syst Rev. 2017;4:CD012226.

11 Abdulsalim S, Unnikrishnan MK, Manu MK, Alrasheedy AA, Godman B, Morisky DE. Structured pharmacist-led intervention programme to improve medication adherence in COPD patients: a randomized controlled study. Res Social Adm Pharm. 2018;14(10): 909-14.

12 Mcalister FA, Ye C, Beaupre LA, Rowe BH, Johnson JA, Bellerose D, et al. Adherence to osteoporosis therapy after an upper extremity fracture: a pre-specified substudy of the C-STOP randomized controlled trial. Osteoporos Int. 2019;30(1):127-34. 
13 Chapman AL, Hadfield M, Chapman CJ. Qualitative research in healthcare: an introduction to grounded theory using thematic analysis. J R Coll Physicians Edinb. 2015; 45(3):201-5.

14 Bryant A, Charmaz K. The SAGE handbook of grounded theory. Los Angeles i.e. Thousand Oaks, CA: SAGE Publications; 2010.

15 Watkins D, Gioia D. Mixed methods research. New York: Oxford University Press; 2015.

16 Leske MC, Heijl A, Hussein M, Bengtsson B, Hyman L, Komaroff E. Factors for glaucoma progression and the effect of treatment: the early manifest glaucoma trial. Arch Ophthalmol. 2003;121(1):48.

17 Leidy NK, Vernon M. Perspectives on patient-reported outcomes: content validity and qualitative research in a changing clinical trial environment. Pharmacoeconomics. 2008; 26(5):363-70
18 Eye Diseases Prevalence Research Group; Friedman DS, Wolfs RC, O'Colmain BJ, Klein BE, Taylor HR, West S. et al. Prevalence of open-angle glaucoma among adults in the United States. Arch Ophthalmol. 2004 Apr; 122(4):532-8.

19 Garway-Heath DF, Crabb DP, Bunce C, Lascaratos G, Amalfitano F, Anand N, et al. Latanoprost for open-angle glaucoma (UKGTS): a randomised, multicentre, placebocontrolled trial. Lancet. 2015;385(9975): 1295-304.

20 Shulman N, Cutter G, Daugherty R, Sexton M, Pauk G, Taylor MJ, et al. Correlates of attendance and compliance in the hypertension detection and follow-up program. Control Clin Trials. 1982;3(1):13-27.
21 Yeung M, O'connor SA, Parry DT, Cochrane GM. Compliance with prescribed drug therapy in asthma. Respir Med. 1994;88(1):31-5.

22 Gold DT. Medication adherence: a challenge for patients with postmenopausal osteoporosis and other chronic illnesses. J Manag Care Pharm. 2006 Sep;12(6 Suppl A):S20-8.

23 Ickovics JR, Cameron A, Zackin R, Bassett R, Chesney M, Johnson VA, et al. Consequences and determinants of adherence to antiretroviral medication: results from adult AIDS clinical trials group protocol 370. Antivir Ther. 2002 Sep;7(3):185-93.

24 Malik R, Baker H, Russell RA, Crabb DP. A survey of attitudes of glaucoma subspecialists in England and Wales to visual field test intervals in relation to NICE guidelines. BMJ Open. 2013;3(5):e002067. 